\title{
Investigation of InS-InSe heterojunctions prepared using sulphurization of p-InSe
}

\author{
Z.D. Kovalyuk, V.Y. Duplavyy and O.M. Sydor \\ Chernivtsi Department of the Institute of Materials Science Problems, NAS of Ukraine \\ 5, Iryna Vilde str., 58001 Chernivtsi, Ukraine, Phone: 8 (0372) 52-00-50; e-mail: chimsp@ukrpost.ua
}

\begin{abstract}
InS/ $p$-InSe heterojunctions were obtained by annealing $p$-InSe samples in sulphur vapours. By means of the atomic force microscopy method, topology of InS film surface was investigated. Current-voltage characteristics of the heterojunction were measured, and principal mechanisms of charge transfer were established. The spectrum of relative quantum efficiency of the heterojunction was measured.
\end{abstract}

Keywords: InS, InSe, heterojunction, current-voltage characteristic, annealing.

Manuscript received 12.12.11; revised version received 16.01.12; accepted for publication 26.01.12; published online 29.02.12.

\section{Introduction}

Semiconductor structures based on layered crystals are of great interest because of the possibility to use them as radiation-resistant photodevices [1]. There are some ways to form heterojunctions based on InSe. As a result of anisotropy of chemical bonds in layered crystals, there is a possibility to obtain plane-parallel plates, the surface of which contains a low density of dangling bonds. The thickness of these plates can be several micrometers thick, what enables to prepare heterostructures (HS) by using the method of direct optical contact [2]. Intrinsic oxide-InSe heterostructures were prepared by annealing indium monoselenide samples in presence of oxygen [3].

In [4] it was established a possibility for $\mathrm{HS}$ preparation by a long-term $(24 \ldots 120 \mathrm{~h})$ annealing in sulphur vapours. The purpose of this paper is to investigate the possibility to create $n$-InS/ $p$-InSe HS by sulphurization of $p$-InSe samples for $12 \mathrm{~h}$. Like to that in [4], we established that parameters of the structure prepared on the basis of $p$-InSe are much better than in the case of $n$-InSe, only $p$-type material being used as substrates.

\section{Experimental}

Initial single crystals InSe were grown using the vertical Bridgman method from a non-stoichiometric composition $\mathrm{In}_{1.03} \mathrm{Se}_{0.97}$. To obtain $p$-type conductivity, indium selenide was doped with $\mathrm{Cd}$ impurity. When its content in the melt reaches $0.01 \%$, the grown crystals have the concentration of holes $p \approx 10^{13} \mathrm{~cm}^{-3}$ and their mobility $\mu \approx 80 \mathrm{~cm}^{2} / \mathrm{V} \cdot \mathrm{s}$ at $T=300 \mathrm{~K}$.
Samples were cleaved from the ingot with a razor blade. Samples for annealing were $0.8 \ldots 1 \mathrm{~mm}$ thick and $2 \times 5 \mathrm{~mm}$ in size.

The annealing was carried out in an evacuated silica ampoule with InSe-substrates and crystalline sulphur located in its opposite parts. The ampoule was placed into the two-region furnace, the temperature in the sulphur location was $550 \mathrm{~K}$, and in the $p$-InSe location $-465 \mathrm{~K}$. Annealing time was equal to $12 \mathrm{~h}$. After the annealing, an $n$-InS film was formed on the surface of InSe. After cleaving and cutting the samples, the obtained HS were $5 \times 5 \times 0.1 \mathrm{~mm}$ in size.

For studying the surface topology, the atomic-force microscopy (AFM) method was used. It enabled to obtain the data about the surface relief in the nanometer scale. Measurements were carried out using the atomicforce microscope Nanoscope IIIa Dimension 3000SPM (Digital Instruments) in the tapping mode.

Current-voltage $(I-V)$ characteristics were measured using the "Solartron 1255" device, and an analysis of the obtained dependences was made by means of the program "CView 3.10".

The photosensitivity spectra were measured at room temperature using the $\mathrm{MDR}-2$ monochromator with the resolution $26 \AA$. The samples were illuminated from the film's side. All the spectra were normalized with respect to the amount of incident photons.

\section{Results and discussion}

Fig. 1 shows three- (a) and two-dimensional (b) AFM images of the obtained InS surface topology, which shows that the surface of the film is formed by spherical nanoobjects $(75 \mathrm{~nm}$ in height and $100 \ldots .200 \mathrm{~nm}$ in diameter). Fig. 1c shows the distribution of heights of nanograins over the lateral plane of thin film (along the line in Fig. 1b). 


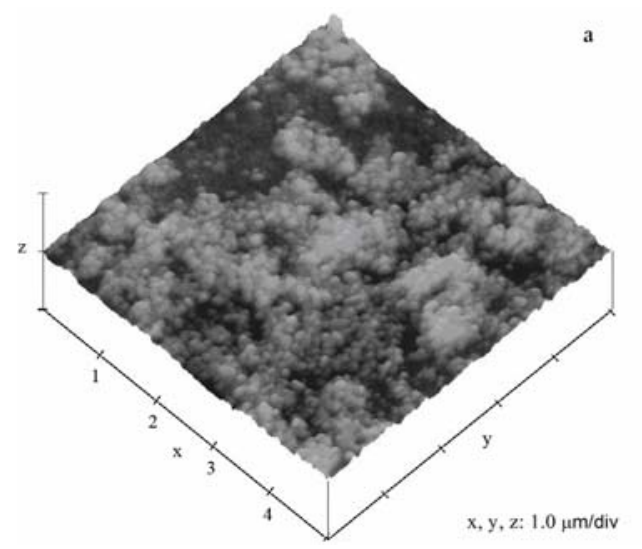

a

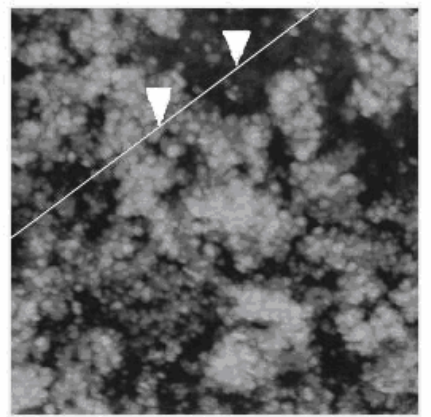

$\mathrm{b}$

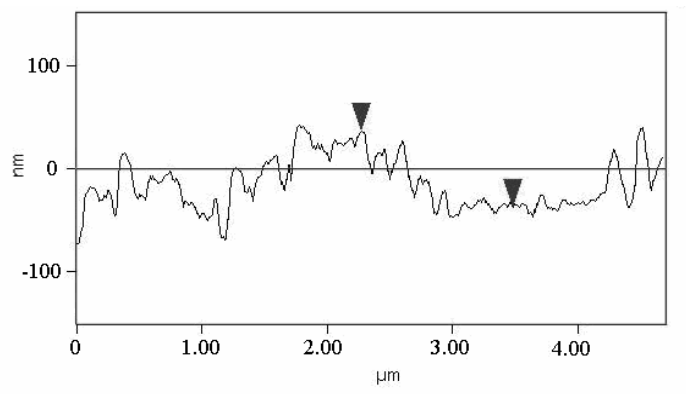

c

Fig. 1. Three-dimensional (a) and two-dimensional (b) AFM images of InS surface, (c) - distribution of nanograin heights along the line of image (b).

In order to determine the change transport mechanism through the heterojunctions under consideration, the $I-V$ characteristics were measured at forward and reverse biases. Fig. 2a shows forward branches of the $I-V$ characteristics at various temperatures in semilogarithm scale. The initial sections of the $I-V$ characteristics at relatively low forward biases are described by the expression

$$
J=J_{s}\left[\exp \left(\frac{q V}{n k T}\right)-1\right],
$$

where $q$ is the elementary charge, $T$ - temperature, $k-$ Boltzmann constant, $n$ - diode ideality factor, $V-$ voltage, and $J_{s}$ - saturation current. At forward biases $V>0.35 \mathrm{~V}, I-V$ characteristics deviate from the expression (1).

The main reason of this deviation is the high series resistance $R_{s}$ of the substrate, as a result voltage mainly drops on the series resistance. In order to take into account the effect of the series resistance on the $I-V$ characteristics, they were plotted in coordinates $\lg J$ vs $V-R_{S} J$. The values of $R_{s}$ were determined by means of extrapolation of the saturated segment of the $R_{\text {diff }}(V)$ dependence within the temperature range from 253 to 332 K. Further, using these plots the diode factor $n$ was calculated using the expression

$n=(q / k T)(\Delta V / \Delta \ln J)$.

Within the temperature range 253 to $293 \mathrm{~K}$, the diode factor $n$ is equal to 2.2, the current transport mechanism in the space-charge region has a tunnel-recombination nature, and at $332 \mathrm{~K}$ the over barrier emission is a dominating current transport.
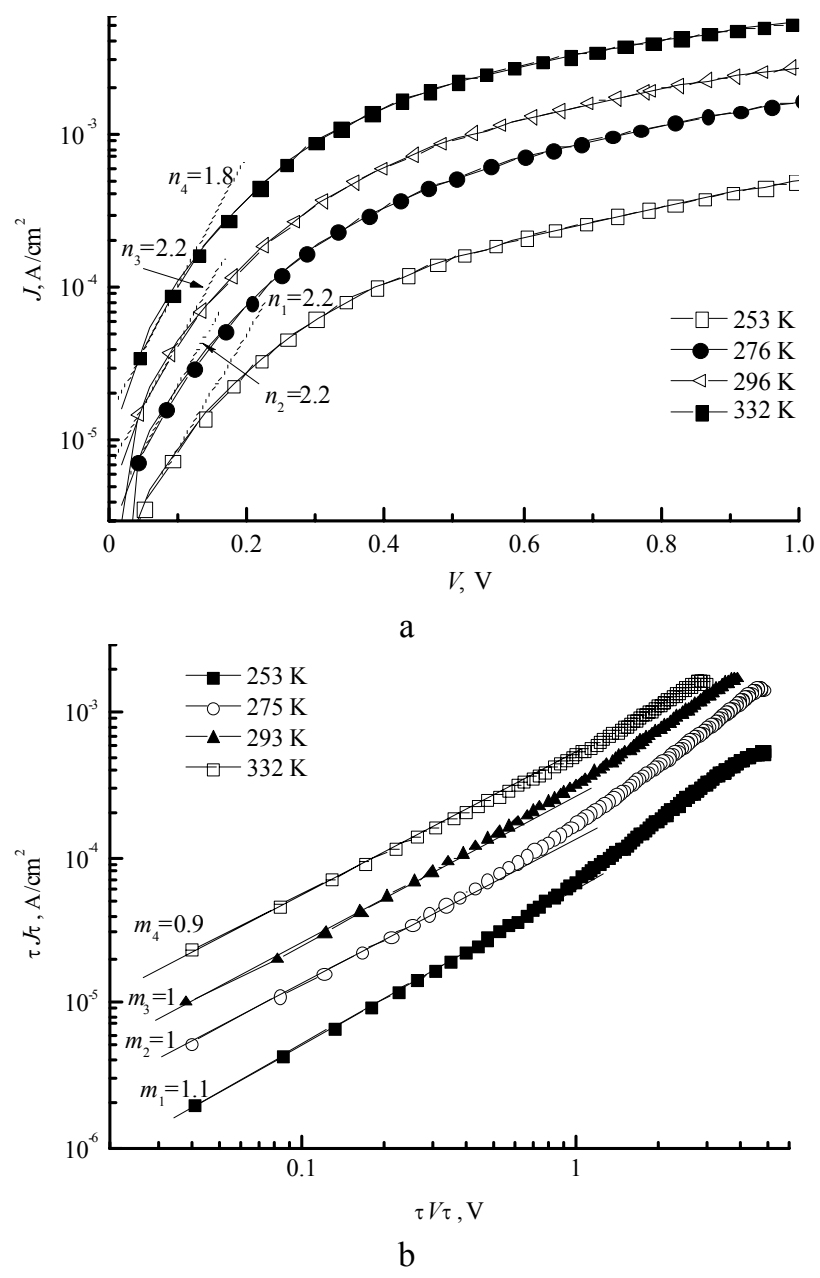

Fig. 2. Forward (a) and reverse (b) branches of the currentvoltage characteristics of $n$-InS/p-InSe heterostructure at various temperatures. 


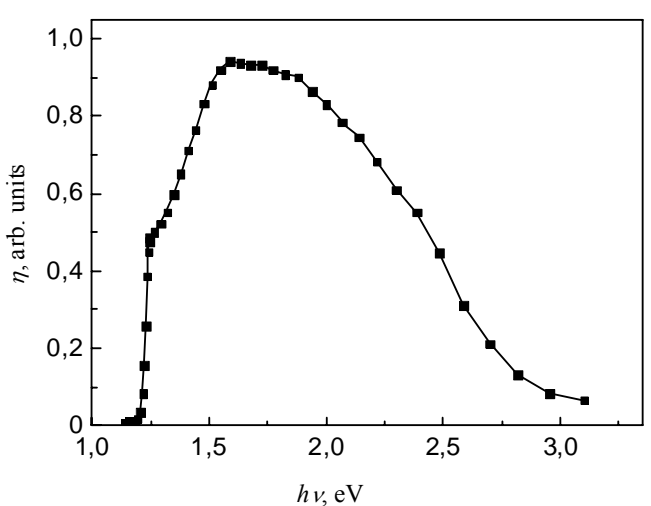

Fig. 3. Spectral dependence of the relative quantum efficiency of photoconversion in the $n$-InS/p-InSe heterostructure.

The reverse branch of the $I-V$ characteristic is governed by the power law. The exponential $m(0.9 \ldots 1)$ is temperature independent due to the space charge limited current mechanism.

The obtained spectra of relative quantum efficiency of $n-\mathrm{InS} / p$-InSe are characterized by a typical shape in the case of heterojunctions. They are limited from both shortwave $(2.8 \mathrm{eV})$ and longwave $(1.2 \mathrm{eV})$ regions (Fig. 3).

\section{Conclusion}

The photosensitive HS InS-InSe was formed by means of the annealing of InSe substrates in sulphur vapours for 12 hours. The obtained thin films possessed the nanoscale structure. The effect of the series resistance on the $I-V$ characteristics was taken into account. The tunnelling-recombination processes were established to be the dominating current transport mechanism within the temperature range from 253 to $294 \mathrm{~K}$. The over barrier emission is the dominating current mechanism at $332 \mathrm{~K}$.

\section{References}

1. Z.D. Kovalyuk, V.N. Katerinchuk, O.A. Politanska, O.N. Sydor and V.V. Homiak, Influence of $\gamma$ irradiation on properties of InSe photodiodes // Pis'ma zhurnal tekhnich. fiziki, 31(9), p. 1-6 (2005), in Russian.

2. V.L. Bakumenko, Z.D. Kovalyuk, L.N. Kurbatov, V.G. Tagaev and V.F. Chishko, Investigation of InSe-GaSe heterojunctions obtained by optical contact // Fizika tekhnika poluprovodnikov, 14 (6), p. 1115-1119 (1980), in Russian.

3. Z.D. Kovalyuk, O.N. Sydor, V.N. Katerynchuk and V.V. Netyaga, Investigation of isotype photosensitive (intrinsic oxide) $n$-InSe heterostructures prepared by long-term thermal oxidation // Fizika tekhnika poluprovodnikov, 41 (9), p. 1074-1077 (2007), in Russian.

4. Z.D. Kovalyuk, O.I. Kushnir, O.N. Sydor, and V.V. Netyaga, Heterostructures manufactured by annealing of InSe single crystals in sulphur vapours // Tekhnologiya konstruirovaniye elektronnoi apparatury, 79(1), p. 61-62 (2009), in Russian.

5. M. Lampert, P. Mark, Current Injection in Solids, Academic Press, New York and London, 1970. 